Supporting Information

\title{
A Biomimetic Approach to Facilitate High Filler Content in Free-Standing and Flexible Thermoelectric Polymer Composite Films Based on PVDF and $\mathrm{Ag}_{2} \mathrm{Se}$ Nanowires
}

Hongju Zhou, ${ }^{a}$ Zhenjie Zhang, ${ }^{a}$ Chengxiao Sun, ${ }^{a}$ Hua Deng, ${ }^{* a}$ Qiang Fu ${ }^{* a}$

${ }^{\text {a }}$ College of Polymer Science and Engineering, State Key Laboratory of Polymer Materials Engineering, Sichuan University, Chengdu 610065, China

* Corresponding author.

Email: huadeng@scu.edu.cn (H. Deng), qiangfu@scu.edu.cn (Q.Fu) 

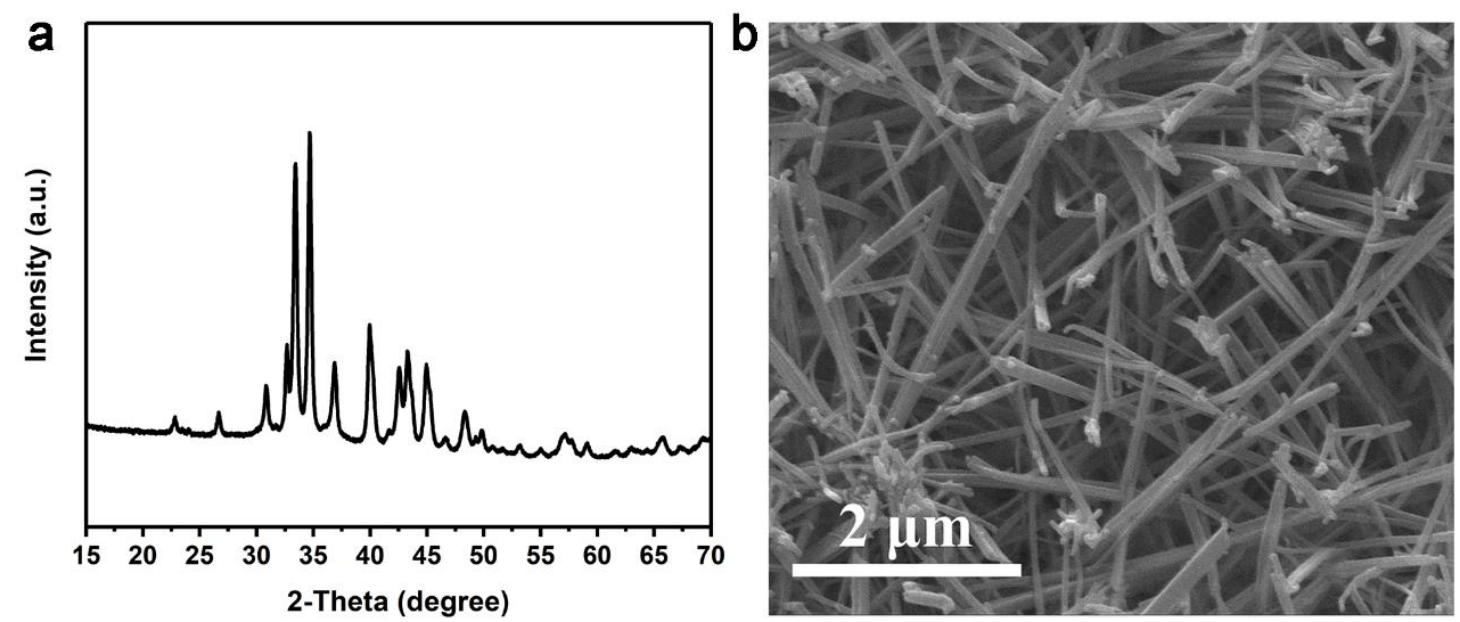

Figure S1. XRD pattern and SEM image of $\mathrm{Ag}_{2} \mathrm{Se}$ NWs.
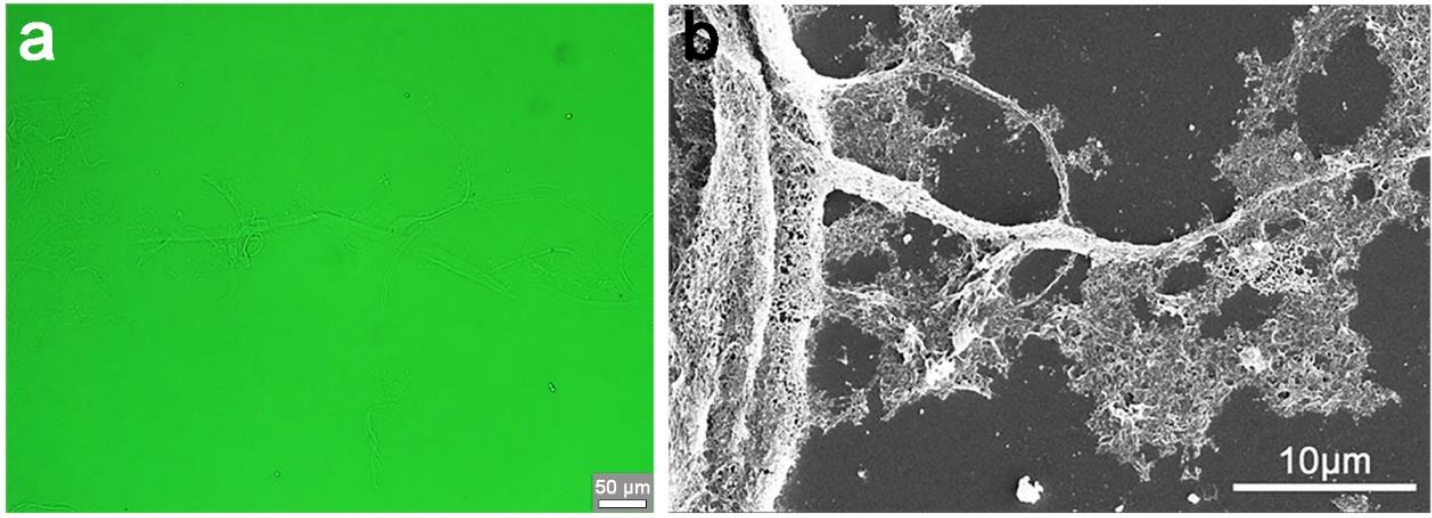

Figure S2. Optical microscopy and SEM image of PVDF dendricolloids. 

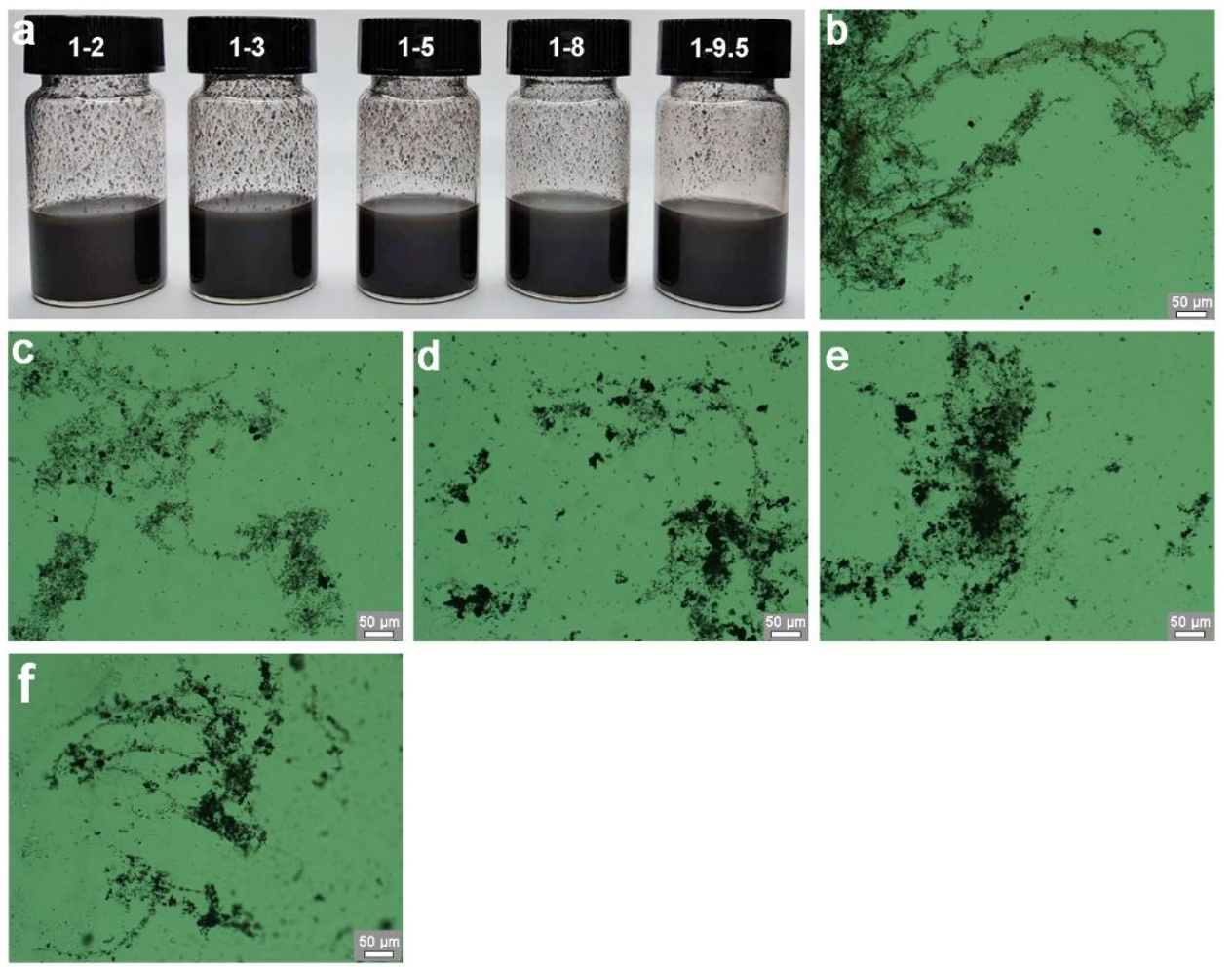

Figure S3. Digital photos (a) and optical microscopy of PVDF/Ag $2 \mathrm{Se}$ composite dispersion samples 1-2 (b), 1-3 (c), 1-5 (d), 1-8 (e) and 1-9.5 (f).
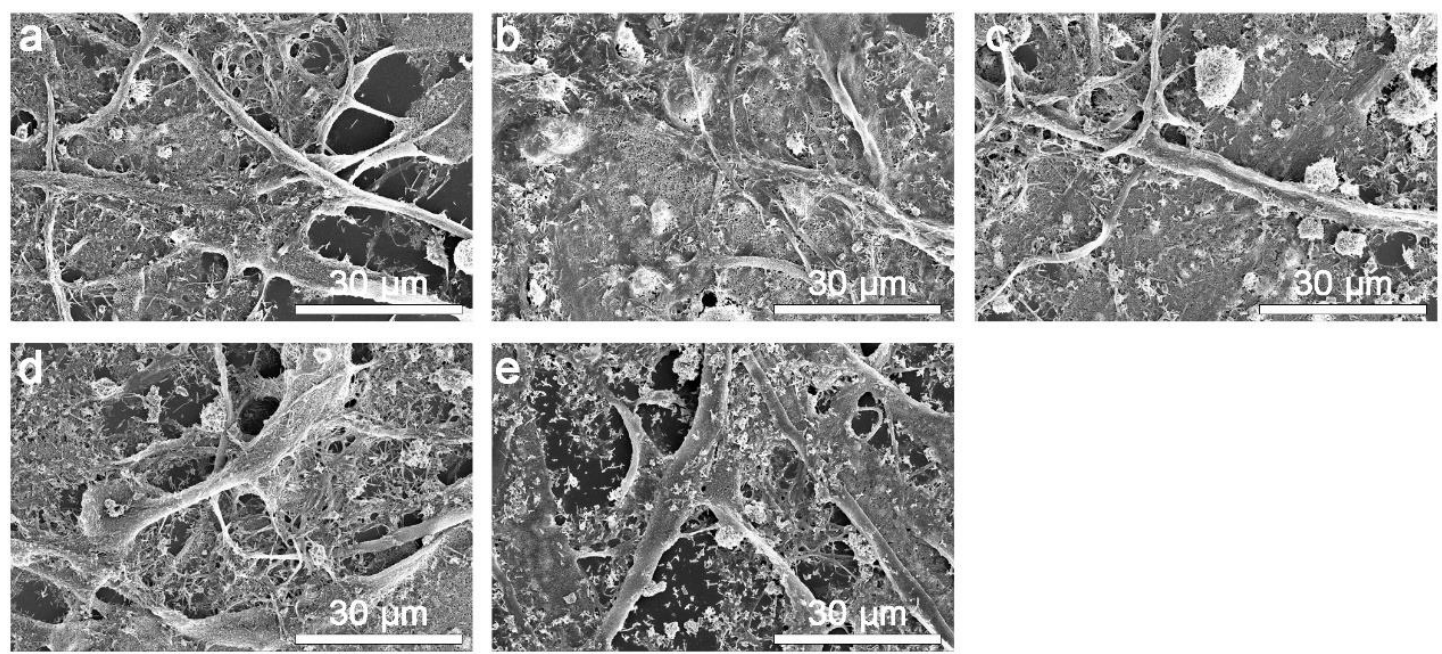

Figure S4. SEM images of PVDF/Ag 2 Se composite dispersion samples 1-2 (a), 1-3

(b), 1-5 (c), 1-8 (d), 1-9.5 (e). 

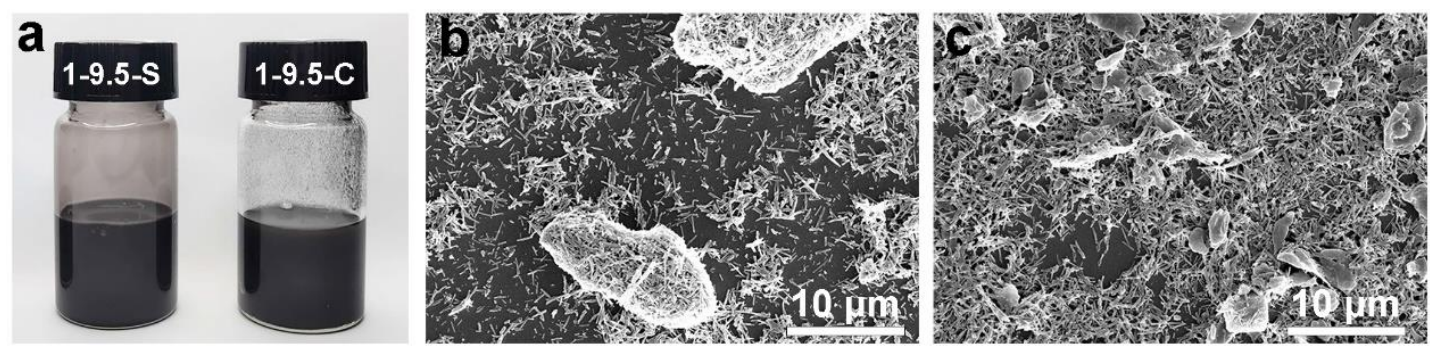

Figure S5. Digital photos (a) and SEM images of sample 1-9.5-S (b) and 1-9.5-C (c).

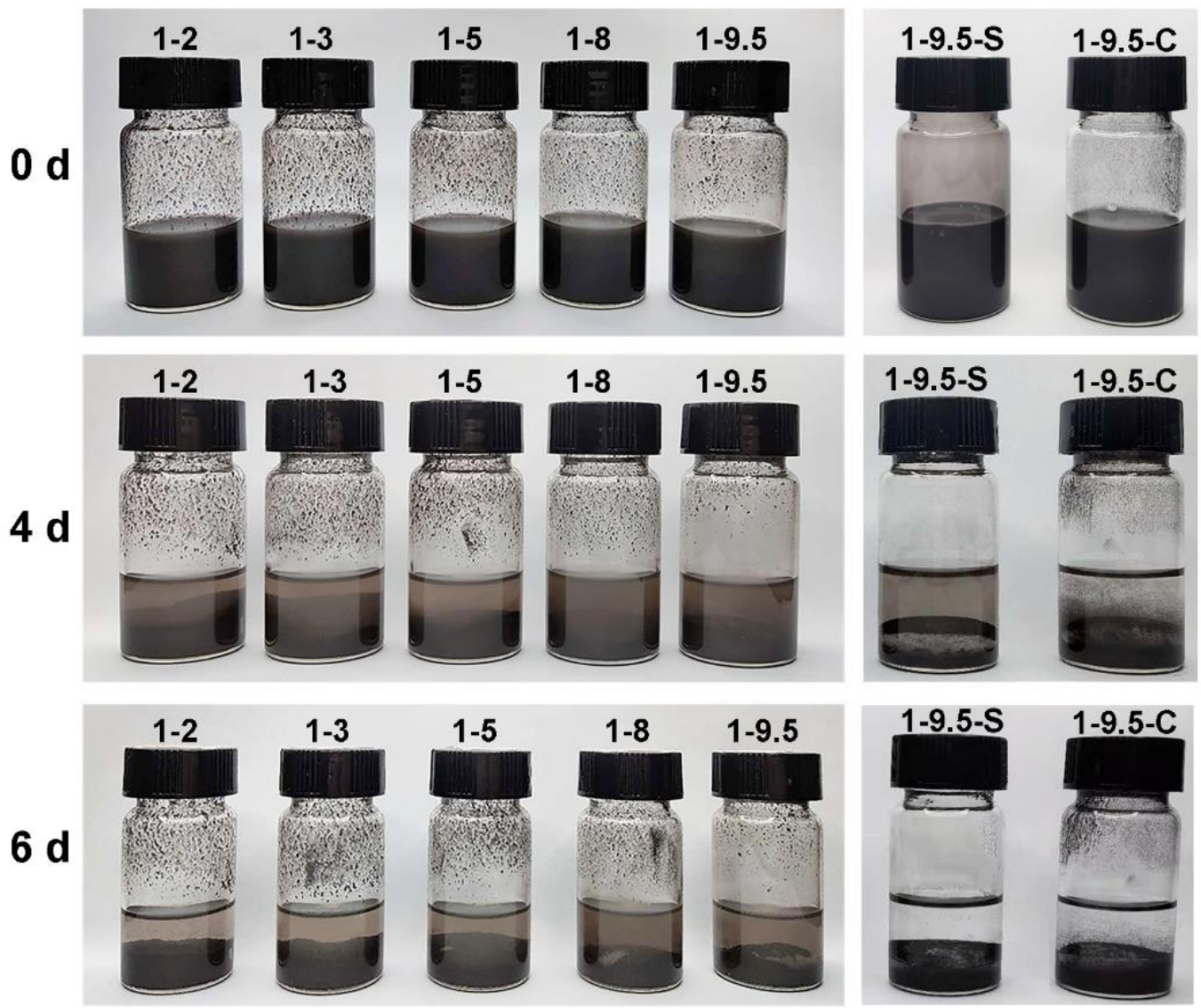

Figure S6. Digital photos of the PVDF/Ag $\mathrm{P}_{2} \mathrm{Se}$ composite suspension 1-2, 1-3, 1-5, 18, 1-9.5, 1-9.5-S and 1-9.5-C (in different columns) originally, after four days and six days, respectively. 

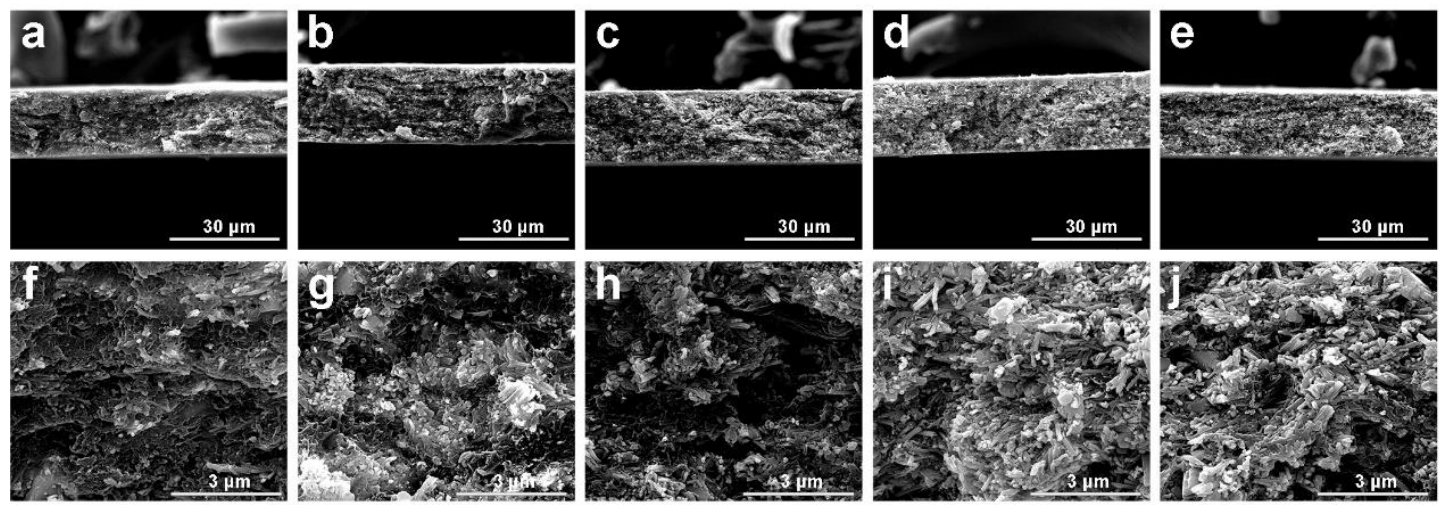

Figure S7. Fraction SEM images of PVDF/ $\mathrm{Ag}_{2} \mathrm{Se}$ composite films samples 1-2 (a, f),

1-3 (b, g), 1-5 (c, h), 1-8 (d, i) and 1-9.5 (e, j) with different magnification.
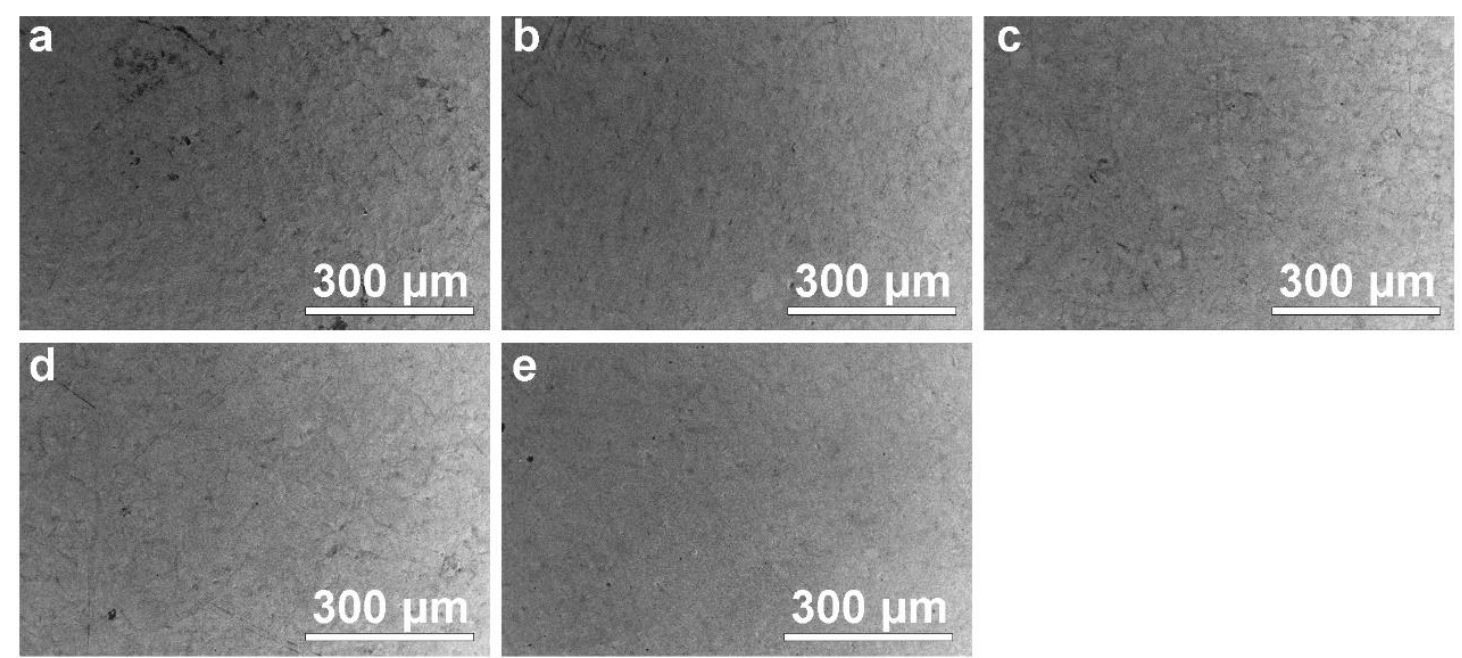

Figure S8. SEM images of PVDF/Ag ${ }_{2} \mathrm{Se}$ composite films surface for sample 1-2 (a),

1-3 (b), 1-5 (c), 1-8 (d) and 1-9.5 (e). 

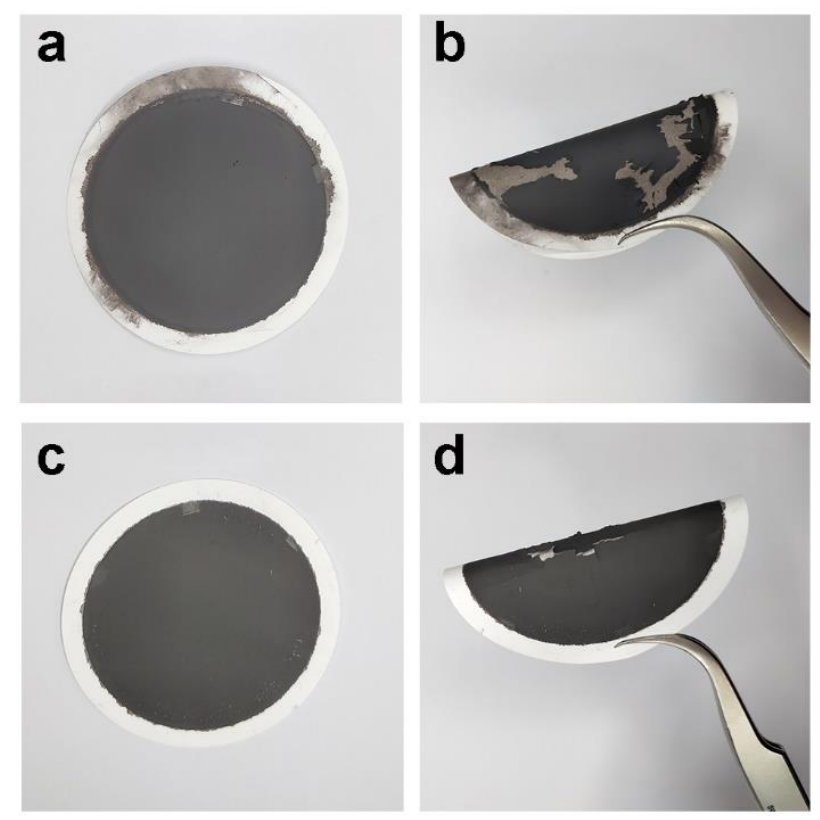

Figure S9. Digital photos of $\mathrm{PVDF} / \mathrm{Ag}_{2} \mathrm{Se}$ films fabricated from sample 1-9.5-S (a, b) and $1-9.5-\mathrm{C}(\mathrm{c}, \mathrm{d})$.

Table S1. Specific heat capacity, thermal diffusivity, experimental density and theoretical density of PVDF/Ag 2 Se composite films.

\begin{tabular}{cccccc}
\hline Sample & $\begin{array}{c}\text { Specific heat } \\
\text { capacity } \\
\left(\mathrm{J} \mathrm{g}^{-1} \mathrm{~K}^{-1}\right)\end{array}$ & $\begin{array}{c}\text { In-plane } \\
\text { thermal diffusivity } \\
\left(\mathrm{mm}^{2} \mathrm{~s}^{-1}\right)\end{array}$ & $\begin{array}{c}\text { Out-of-Plane } \\
\text { thermal diffusivity } \\
\left(\mathrm{mm}^{2} \mathrm{~s}^{-1}\right)\end{array}$ & $\begin{array}{c}\text { Experimental } \\
\text { Density } \\
\left(\mathrm{g} \mathrm{cm}^{-3}\right)\end{array}$ & $\begin{array}{c}\text { Theoretical } \\
\text { Density } \\
\left(\mathrm{g} \mathrm{cm}^{-3}\right)\end{array}$ \\
\hline $1-2$ & 0.5947 & $3.593 \pm 0.141$ & $0.0322 \pm 0.0112$ & 3.27 & 3.73 \\
$1-3$ & 0.4965 & $3.676 \pm 0.170$ & $0.0334 \pm 0.0050$ & 3.73 & 4.32 \\
$1-5$ & 0.3983 & $4.343 \pm 0.084$ & $0.0390 \pm 0.0057$ & 4.04 & 5.13 \\
$1-8$ & 0.3329 & $4.730 \pm 0.103$ & $0.0470 \pm 0.0057$ & 4.47 & 5.86 \\
$1-9.5$ & 0.3142 & $4.719 \pm 0.114$ & $0.0460 \pm 0.0081$ & 4.82 & 6.13 \\
\hline
\end{tabular}




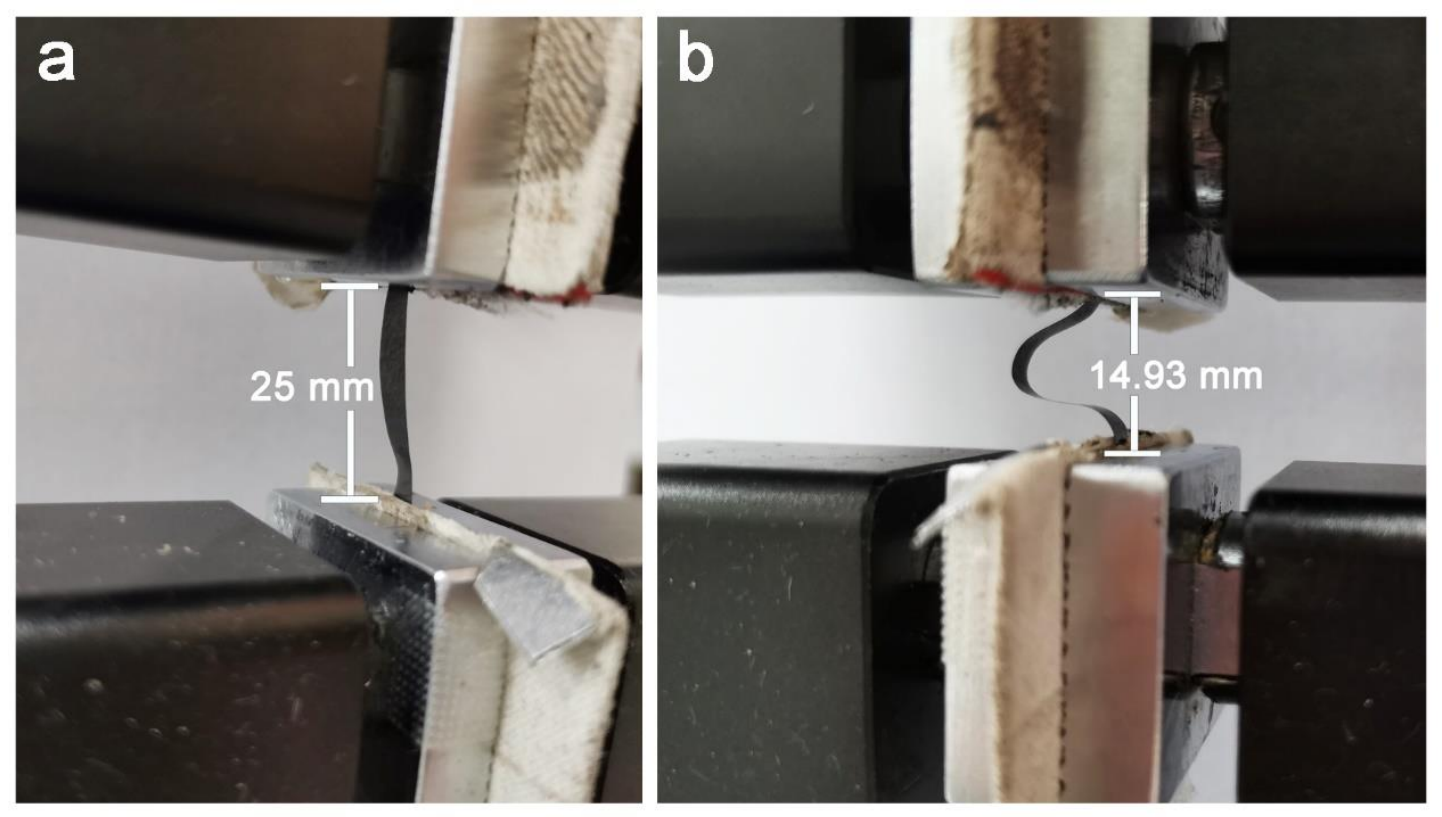

Figure S10. Digital photos of a sample 1-9.5 strip (3 mm in width) before (a) and after (b) bending to $40 \%$ strain.

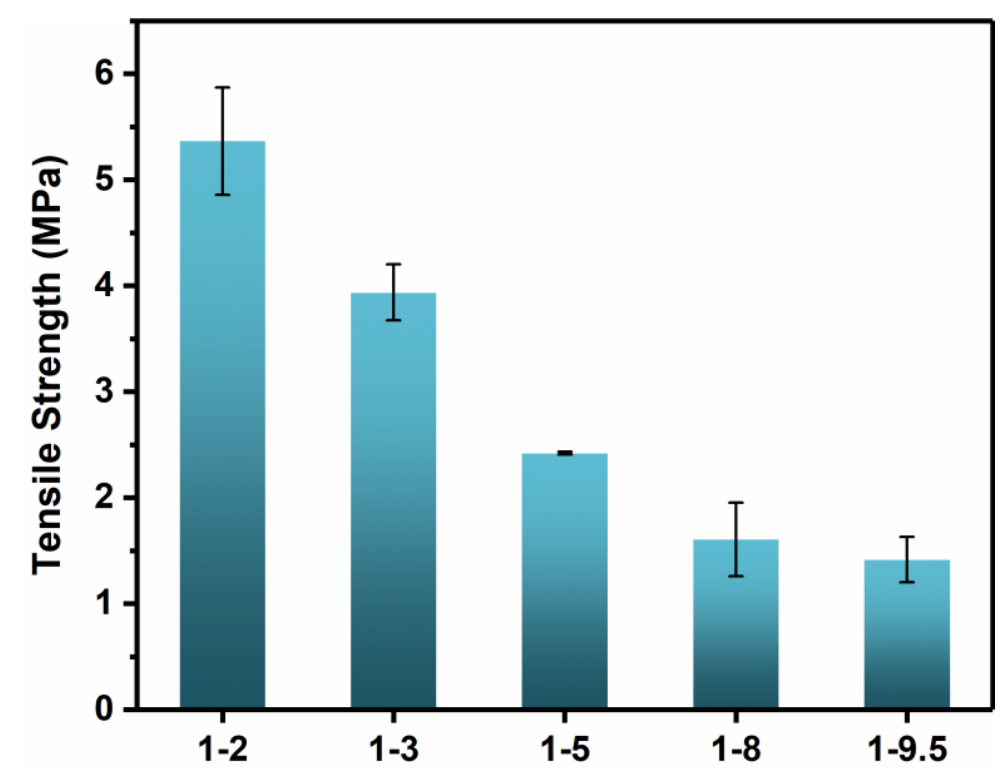

Figure S11. Tensile strength of PVDF/ $\mathrm{Ag}_{2} \mathrm{Se}$ composite films at room temperature. 